\title{
Chimera-type states induced by local coupling
}

\author{
M. G. Clerc, ${ }^{1}$ S. Coulibaly, ${ }^{2}$ M. A. Ferré, ${ }^{3}$ M. A. García-Ñustes, ${ }^{3}$ and R. G. Rojas ${ }^{3}$ \\ ${ }^{1}$ Departamento de Física, Facultad de Ciencias Físicas y Matemáticas, Universidad de Chile, \\ Casilla 487-3, Santiago, Chile \\ ${ }^{2}$ Laboratoire de Physique des Lasers, Atomes et Molécules, CNRS UMR 8523, Université de Lille 1 Sciences et Technologies, \\ 59655 Villeneuve d'Ascq Cedex, France \\ ${ }^{3}$ Instituto de Física, Pontificia Universidad Católica de Valparaíso, casilla 4059, Valparaíso, Chile \\ (Received 15 April 2015; revised manuscript received 25 October 2015; published 5 May 2016)
}

\begin{abstract}
Coupled oscillators can exhibit complex self-organization behavior such as phase turbulence, spatiotemporal intermittency, and chimera states. The latter corresponds to a coexistence of coherent and incoherent states apparently promoted by nonlocal or global coupling. Here we investigate the existence, stability properties, and bifurcation diagram of chimera-type states in a system with local coupling without different time scales. Based on a model of a chain of nonlinear oscillators coupled to adjacent neighbors, we identify the required attributes to observe these states: local coupling and bistability between a stationary and an oscillatory state close to a homoclinic bifurcation. The local coupling prevents the incoherent state from invading the coherent one, allowing concurrently the existence of a family of chimera states, which are organized by a homoclinic snaking bifurcation diagram.
\end{abstract}

DOI: 10.1103/PhysRevE.93.052204

Coupled oscillators under the influence of injection and dissipation of energy exhibit a rich spatiotemporal dynamics [1-3], such as phase turbulence [1], synchronization [2], defects turbulence [4], random occurrence of coherence events [5], defect mediated turbulence [6], spatiotemporal intermittency [7], and quasiperiodicity in extended system [8]. In the past decade, a phenomenon that has received a great deal of attention are spatiotemporal patterns of coexisting coherent and incoherent behavior, known as chimera states [9]. The first observation of chimera state was made by Kuramoto and Battogtokh in the complex Ginzburg-Landau equation with a weak nonlocal coupling [10]. Subsequently, it has been investigated in a variety of models like phase oscillators [911], chemical oscillators [12], and planar oscillators [13]. It was shown that they are not limited to phase oscillators but can be found in a large variety of different systems, including time-discrete maps [14], neuron models [15], time-continuous chaotic models [16], and networks [17,18]. Recently, the chimera states have been reported experimentally in a chemical system [19], an optoelectronic setup [20], and a mechanical oscillator network [21]. Therefore, coupled discrete systems showing coexistence of incoherent and coherent states, chimera-type states, are ubiquitous in nature. The chimera states are apparently peculiar to the intermediate case of nonlocal coupling [9-11]; however, recently it was shown that it is possible to find in systems with strong nonlocal limit [22] and global coupling [18]. Turbulent chimera states in extended systems with long range or mean field coupling have been reported $[18,23]$ and intermittent chaotic chimeras in oscillators with inertia have been recently reported for two populations of oscillators [24]. The establishment of chimeratype states in purely local coupling-systems that only consider interaction between adjacent neighbors-previously is not expected because nonlocal interaction was considered essential. Recently, in reaction diffusion coupled systems with more than one variable and different dynamical temporal scales (fast and slow) chimera states have been observed in one [25] and two dimensions [26].
The aim of this paper is to investigate the formation of chimera-type states in locally coupled systems without different time scales. Based on a simple model of coupled nonlinear oscillators, an extended version of the BogdanovTakens normal form [27], we have identified the necessary requirements for chimera states formation. Close to a homoclinic bifurcation, this model exhibits a coexistence of an incoherent extended oscillatory state and a steady uniform one. As a result of the weak local coupling, a locking phenomenon takes place, inhibiting the propagation of fronts connecting the two states, forming a motionless chimera state (cf. Fig. 1). Depending on initial conditions, a family of chimera states can appear and disappear, following a homoclinic snaking bifurcation diagram [28]. In the continuum limit, the chimera-type states are not a robust solution.

Let us introduce a simple model of locally coupled oscillators with nonlinear damping (extended Bogdanov-Takens normal form [27])

$$
\begin{aligned}
\ddot{u}_{i}(t)= & -u_{i}-a u_{i}^{2}-u_{i}^{3}+\left(\mu-\delta u_{i}^{2}\right) \dot{u}_{i} \\
& +\Delta\left(u_{i+1}-2 u_{i}+u_{i-1}\right)+\kappa\left(\dot{u}_{i+1}-2 \dot{u}_{i}+\dot{u}_{i-1}\right),
\end{aligned}
$$

with $i=\{0,1, \ldots, N\}$, and Neumann-type boundary conditions, i.e., $u_{-1} \equiv u_{1}, \dot{u}_{-1} \equiv \dot{u}_{1}, u_{N+1} \equiv u_{N-1}$, and $\dot{u}_{N+1} \equiv$ $\dot{u}_{N-1} \cdot u_{i}(t)$ is the position of $i$ oscillator. The first term on the right side stands for Hooke's law, while the second and third are the nonlinear response of the oscillator. The fourth term accounts for the nonlinear damping. The last two terms describe the dispersive and the diffusive coupling, respectively, where $\Delta$ and $\kappa$ account for coupling strength. For $\Delta=\kappa=0$, model (1) reduces to the Bogdanov-Takens normal form for an elementary oscillator [29]. Using the Hilbert transform [30], one can obtain the phase $\left(\varphi_{i}\right)$ and amplitude of the oscillators. Figure 1 shows one of the typical chimera-type states observed in model (1). The observed state is obtained for large enough time compared with the inverse of the largest Lyapunov exponent. The coherent state corresponds to a steady state of 


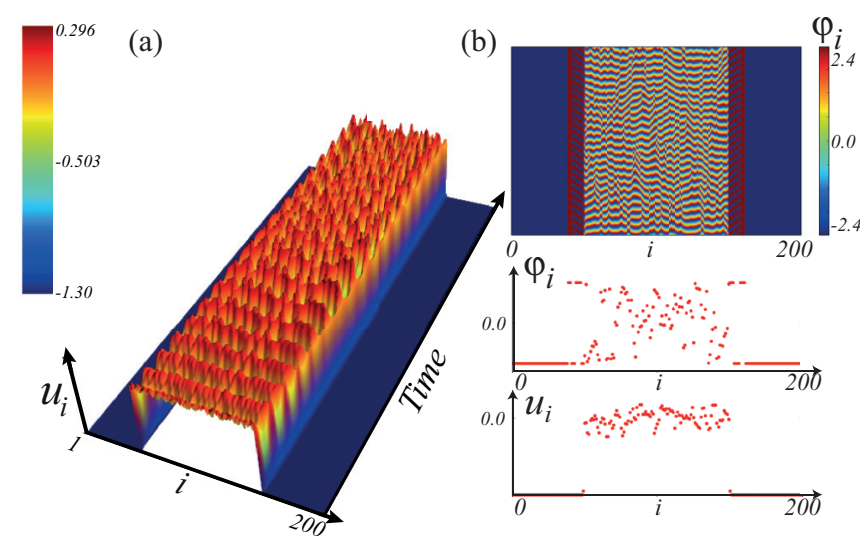

FIG. 1. Spatiotemporal evolution of chimera-type state obtained from model (1) with $\Delta=0.04, \mu=0.05, a=2.07, \kappa=0.04$, and $\delta=1$. (a) Spatiotemporal evolution of $u_{i}$ oscillators. (b) Spatiotemporal evolution of respective phase $\varphi_{i}$ obtained using the Hilbert transform. The insets show position and phase profiles of the oscillators in a given time.

the oscillators, conversely, the incoherent state is a permanent spatiotemporal chaotic state, as we will show later. Note that the phase incoherence region is larger in comparison to that of the incoherence oscillator positions.

Considering different initial conditions the system exhibits a family of chimera-type states that coexist in the same range of parameters (cf. Fig. 2). All numerical simulations were conducted using Runge-Kutta fourth-order algorithm. The initial amount of oscillators in the incoherent state will determine which of the possible solutions will appear. Thus each chimera-type mainly has a different width. To provide a parameter that characterizes each solution of the family, we introduce the mean relative area of the oscillators, $\|u\| \equiv$ $\left(\int_{0}^{T} d t \sum_{i} u_{i}(t)-u^{+}\right) / N T$ for a large time $T$, where $u^{+}=$

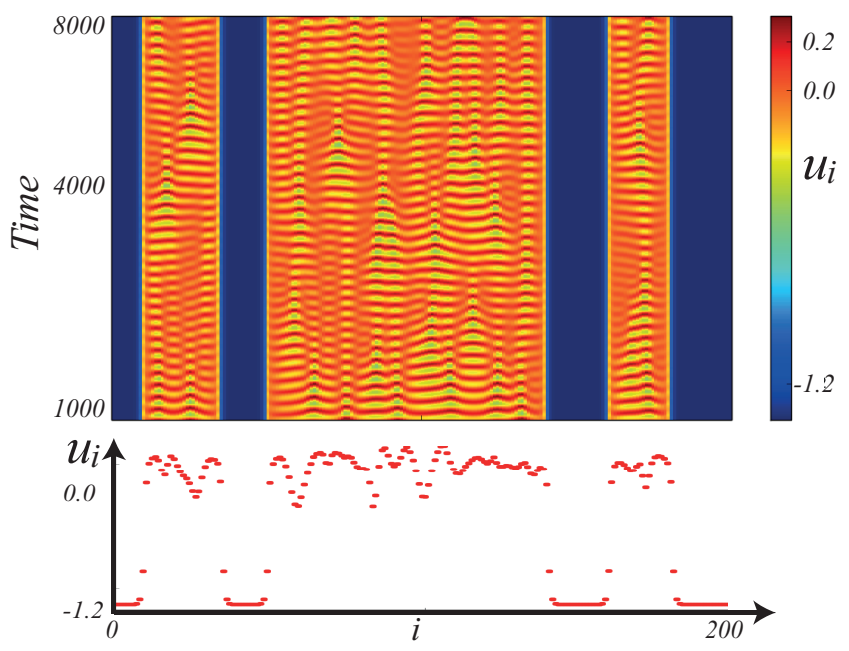

FIG. 2. Spatiotemporal evolution of different chimera-type states obtained from Eq. (1) with $\Delta=0.04, \mu=0.05, a=2.05, \kappa=$ 0.04 , and $\delta=1$. The bottom panel shows the profile of the position of the oscillators in a given time.

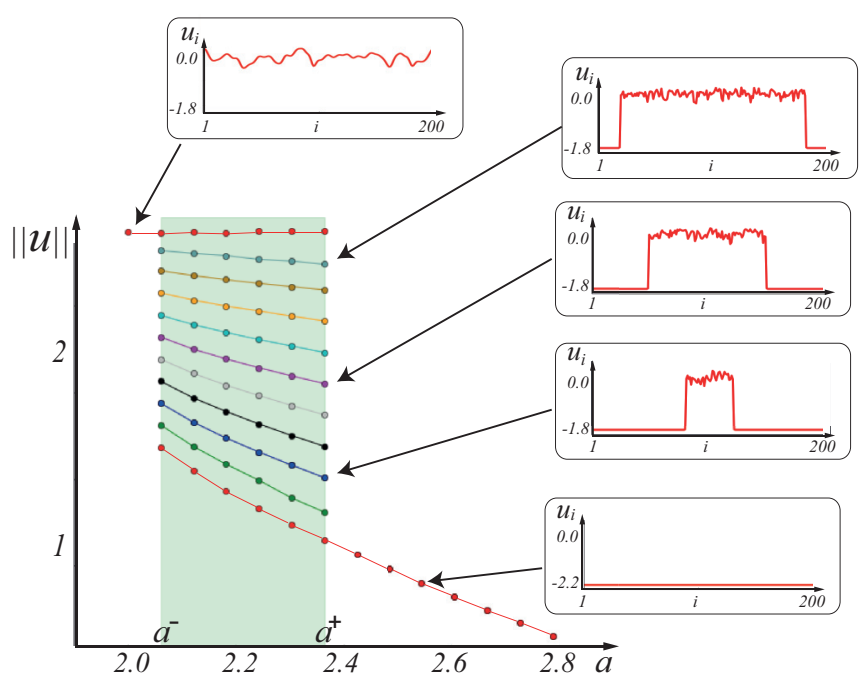

FIG. 3. Numerical homoclinic snaking bifurcation diagram of chimera states with respect to the nonlinearity parameter $a$ of Eq. (1) with $\Delta=0.06, \mu=0.05, \kappa=0.06$, and $\delta=1$. Numerical simulations consider 200 coupled oscillators. The painted area accounts for coexistence region of chimera states, $\left\{a^{-}, a^{+}\right\}=\{2.06,2.36\}$. Points represent the time mean area under the curve by oscillator. The curves inside painted area correspond to changes in the mean relative area of chimera states as function of nonlinearity parameter $a$. These states appear and disappear by a sequence of saddle-node bifurcations. The insets depict some different chimera and the extended states in a given time.

$-\left(a+\sqrt{a^{2}-4}\right) / 2$ is the coherence steady state. Figure 3 displays the bifurcation diagram of chimera-type states with respect to the nonlinearity parameter $a$. The vertical axis accounts for the parameter $\|u\|$. The chimera-type states exhibit a homoclinic snaking bifurcation diagram [28], that is, these states appear and disappear by a sequence of saddle-node bifurcations [31], which occur all around a given value of the nonlinearity parameter in both extremes of the coexistence region. This region and values are depicted by $\left\{a^{-}, a^{+}\right\}$in Fig. 3. Increasing (decreasing) coupling parameter $\Delta$ the coexistence region of different chimeralike states shrinks (expands). This type of bifurcation diagram is typically found for forming patterns systems. This diagram was predicted theoretically in Ref. [28], numerically studied in Refs. [31,32], and subsequently observed experimentally in an optical system [33].

To understand chimera-type state formation in model (1), let us first study the dynamics of an uncoupled oscillator. As we mentioned before, for $\Delta=\kappa=0$, the model describes the dynamics of an elementary oscillator. This oscillator has three fixed points $u^{0}=0$, and $u^{ \pm}=-\left(a \pm \sqrt{a^{2}-4}\right) / 2$. The fixed points $u^{+}$and $u^{-}$are stable and unstable, respectively. These points emerge from a saddle-node bifurcation at $a^{2}=$ $a_{s n}^{2}=4$. Figure 4(a) illustrates the bifurcation diagram of the elementary oscillator as a function of the nonlinearity parameter $a$. The fixed point $u^{0}$ is stable when the coefficient of the linear damping term is negative $(\mu<0)$. This fixed point, as well as in the van der Pol oscillator, undergoes an oscillatory instability-Andronov-Hopf bifurcation-when 


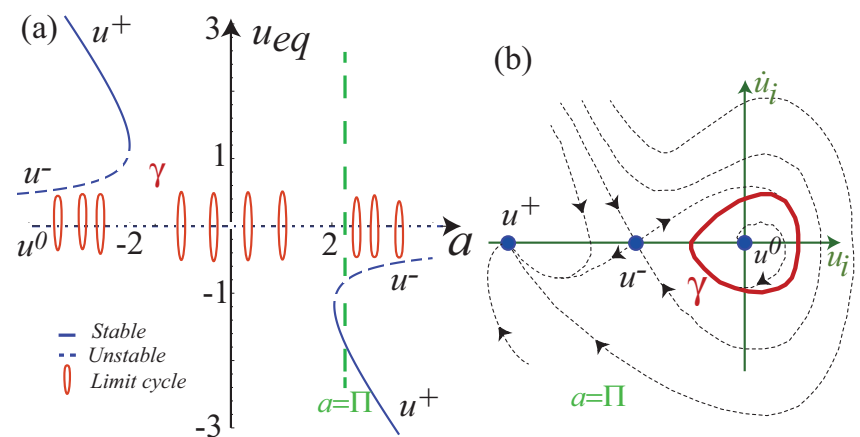

FIG. 4. Properties of elementary oscillators model (1) with $\Delta=$ $\kappa=0$. (a) Schematic bifurcation diagram of oscillator $u_{i}(t)$ as function of nonlinearity parameter $a$; the vertical axis accounts for the value of equilibria states, $u_{e q}$. (b) Phase portrait for $a=\Pi>2$. The equilibrium points and limit cycle are represented by $\left\{u^{+}, u^{-}, u^{0}\right\}$ and $\gamma$, respectively.

the damping coefficient is zero $\left(\mu_{c}=0\right)$. Increasing $\mu$, the oscillator exhibits the emergence of a limit cycle. In Fig. 4(a) we have represented the limit cycles by solid closed curves ( $\gamma$ curves). The phase portrait of the elementary oscillator for $a^{2}>4$ is depicted in Fig. 4(b).

When the oscillators are spatially coupled, the coherent state-related to stable fixed points of the elementary oscillators-is stable and corresponds to a homogeneous steady state. Conversely, when the coupled oscillators are near the homoclinic bifurcation of an elementary oscillator, the synchronization becomes unstable [27]. Given that each oscillator becomes chaotic in this region, an incoherent state is created. To shed light on this mechanism, let us consider the strong local coupling limit of Eq. (1),

$$
\partial_{t t} u=-a u^{2}-u-u^{3}+\left(\mu-\delta u^{2}\right) \partial_{t} u+\tilde{\Delta} \partial_{x x} u+\tilde{\kappa} \partial_{x x t} u,
$$

where the diffusion and dispersion coefficients are, respectively, $\tilde{\Delta}=\Delta d x^{2}$ and $\tilde{\kappa}=\kappa d x^{2}$, with $d x$ denoting an arbitrary infinitesimal quantity. In this limit, the position of the oscillators, $u_{i}(t)$, is promoted to a scalar field $u(x, t)$. The above model have been already used to explain the emergence of spatiotemporal intermittency [27].

Considering the asymptotic change of variable $u(x, t)=$ $A(t) e^{-i t}+\bar{A} e^{i t}-2 a|A|^{2}+a\left(A^{2} e^{2 i t}+\bar{A}^{2} e^{-2 i t}\right) / 3+\cdots$ with $\mu \ll 1$ (close to the Andronov-Hopf bifurcation), the envelope of the oscillation $A(x, t)$ satisfies the complex Ginzburg-Landau equation

$$
\partial_{t} A=\frac{\mu A}{2}-\left[\frac{\delta}{2}-i \Omega\right]|A|^{2} A+\left(\frac{\tilde{\kappa}}{2}-\frac{i \tilde{\Delta}}{2}\right) \partial_{x x} A,
$$

where $\Omega \equiv 3 / 2-5 a^{2} / 3$. Hence the above amplitude equation describes the dynamics of the oscillators around the equilibrium position $u(x, t)=0$ [1]. The solution describing the synchronized oscillations is $A(t)=\sqrt{\mu / \delta} e^{i \mu \Omega t / \delta}$. A linear stability analysis of this solution leads to the following instability condition: $a^{2} \geqslant a_{c} \equiv 3(3+\kappa \delta / \Delta) / 10$, which corresponds to the Benjamin-Feir criteria [1]. In this region of the parameter

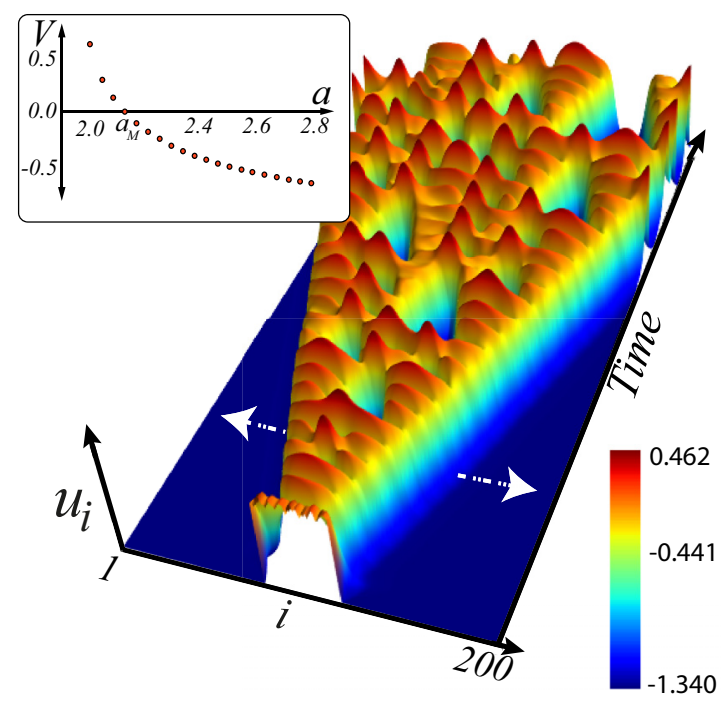

FIG. 5. Front propagation between a spatiotemporal intermittent and a homogeneous state of Eq. (1) with $\Delta=4, \mu=0.25, a=$ $2.08, \kappa=4$, and $\delta=1$. The inset shows the front velocity as a function of nonlinearity parameter $a . a_{M}$ accounts for the Maxwell point.

space this instability leads to the emergence of spatiotemporal intermittency [7]. The expression $\kappa \delta / \Delta$ stands for the relation between the strength of dispersion, diffusion, and nonlinear damping. For coupling strength of order one $(\delta=1, \Delta=2$, and $\kappa=1$ ), the critical nonlinearity parameter takes the value $a_{c}=\sqrt{3 / 2}$. Hence synchronized oscillations become unstable before entering in a region of bistability with the uniform $u^{+}$ state $\left(\left|a_{s n}\right|>\left|a_{c}\right|\right)$. Thus, in bistability region one expects to observe complex fronts between an intermittent and a uniform state. Figure 5 shows the front propagation, using model (1), in the limit of strongly coupled oscillators. For large nonlinearity parameter, the homogeneous state invades the intermittent one. As illustrated in the inset of Fig. 5, front speed can change continuously as a function of parameter $a$. There is only one point in the parameter space where the front speed is zero-the Maxwell point. In Fig. 5, we have noted it with the symbol $a_{M}$. This point stands for that both states are "energetically" equivalent and, therefore, none of the states invade the other one. However, this state is unstable. Therefore, in the strong local coupling limit of model (1), chimera states are not a robust solution.

In Refs. [34,35], they have shown that weak local coupling $(\Delta \sim \kappa \ll 1)$ in dissipative systems causes a propagation failure or pinning of fronts, in a large region of parameters known as pinning range. That is, the fronts connecting different states become motionless. The above phenomenon can be understood by considering a strong local coupling limit with a phenomenological periodic potential forcing, Peierls-Nabarro force, which accounts for the discreteness of the system under study. This potential was introduced in the framework of continuous theory of solid-state physics to understand the dynamics of dislocations in an underlying atomic lattice [36-38]. Therefore, discrete systems from the viewpoint of continuous systems are characterized by having a dynamical evolution 


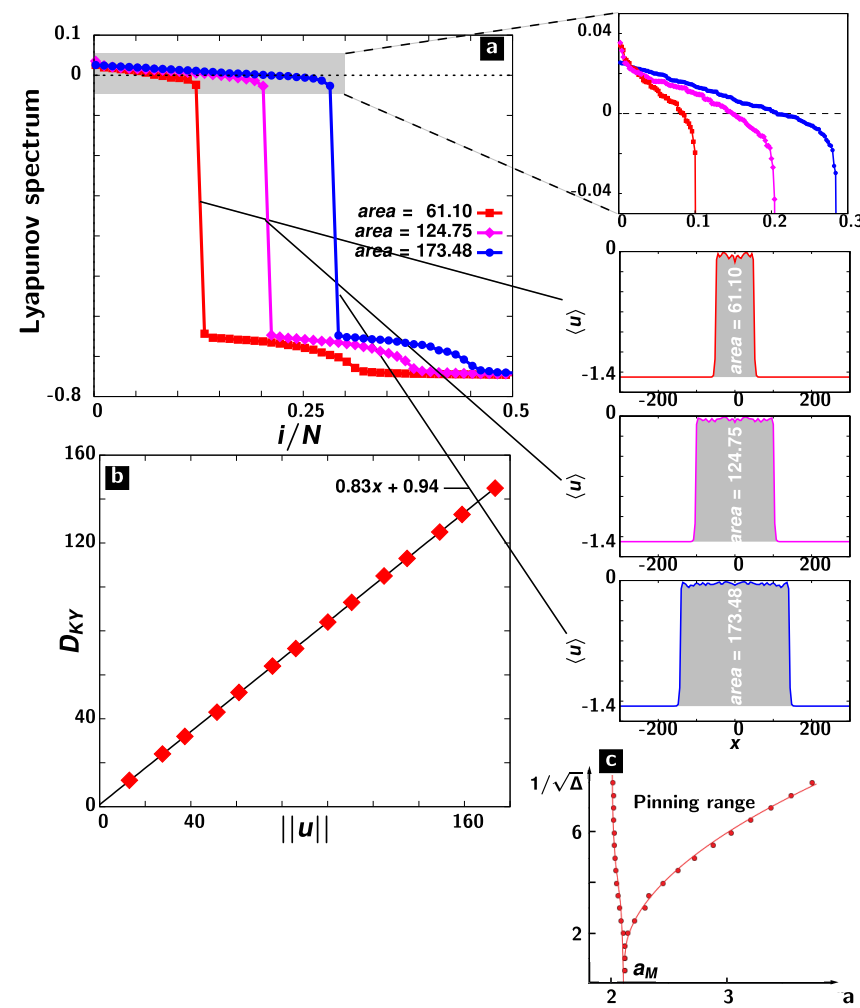

FIG. 6. Properties of chimera states of Eq. (1) with $\Delta=$ $0.06, \mu=0.04, a=2.05, \kappa=0.06$, and $\delta=1$. (a) The Lyapunov spectrum of chimera state. In the inset are represented a magnification of Lyapunov spectrum and chimera states under consideration. (b) The Kaplan-Yorke dimensions as a function of their area with respect to coherent state. (c) Pinning range as a function of nonlinearity parameter and level of intensity coupling; $a_{M} \equiv 3 \sqrt{2} / 2$ accounts for the Maxwell point.

amended by a periodic force that accounts for the effect of discreteness [35]. Hence, considering local weak coupling of nonlinear oscillators in Eq. (1), fronts connecting a uniform and an intermittent state should be locked in a long region of parameters as a result of the discreteness. Thus the system will exhibit chimera-type states. Indeed, the chimera-type states are formed by two pinning fronts (cf. Figs. 1-3). As a result of fronts interaction and local coupling, one expects to observe a family of chimera-type states, which are organized by a homoclinic snaking bifurcation diagram (see Fig. 3) [35]. The distance between fronts - chimera width-characterizes the family of chimera-type states by means of the relative mean area $\|u\|$.

Figure 6(c) shows the pinning region as a function of the level of coupling and nonlinearity parameter $a$. From this graph one concludes that, as the coupling gets stronger, the pinning region decreases and it converges asymptotically to the Maxwell point. Conversely, when one decreases the coupling strength, the pinning region increases. To characterize dynamically, in the limit of weak coupling, we have calculated the Lyapunov spectrum of the chimera-type and their respective Yorke-Kaplan dimension [39]. The Lyapunov spectrum characterizes the exponential separation of trajectories with nearly identical initial conditions in the phase space. Hence a continuous number of positive Lyapunov exponents indicate that the dynamical behavior have a spatiotemporal chaotic nature. The Yorke-Kaplan dimension corresponds to the minimum number of active degrees of freedom that contribute to the chaotic dynamics [39]. Figure 6(a) displays the Lyapunov spectrum for chimera-type states with different mean relative area $\|u\|$. The Lyapunov spectra were computed using the strategy proposed in Ref. [40]. This spectrum clearly shows a separation between unstable and stable modes. Increasing the size of the chimera-type state this separation mode remains. Figure 6(b) shows the Yorke-Kaplan dimension as a function of $\|u\|$, which is a signature of spatiotemporal chaos $[4,41]$. From these outcomes, we infer that the observed chimera-type are composed of a uniform steady state (coherent) and a spatiotemporal chaotic state (incoherent).

The effect of local coupling is responsible for the pinning of fronts between coherent and incoherent state. In the strong local coupling limit, chimera-type states are not a robust solution. In this limit the spatiotemporal chaos or uniform state always invades the other state, except for $a=a_{M}$. Thus one requires a front locking mechanism. For spatiotemporal chaotic patterns coexisting with uniform state, this mechanism can be achieved through the coupling between the envelope and the underlying pattern $[42,43]$, which induces on the front dynamics a periodic forcing. In this context it has been observed experimentally and numerically the spatiotemporal chaotic localized state [44], called chaoticons, which correspond to the continuous counterpart of chimera-type states.

In conclusion, we have investigated the formation requirements for chimera-type states in locally coupled systems without different time scales. They took place in systems that present limit cycles near a homoclinic bifurcation and bistability with a uniform steady state. The homoclinic bifurcation is responsible for the emergence of incoherent oscillations, spatiotemporal chaos. As a result of the local coupling, the incoherent oscillatory state cannot invade the coherent one. Likewise, this allows the system to display a family of chimeratype states, which are organized by a snaking bifurcation diagram. The previous analysis is not a peculiar behavior of the model under study. Incoherent oscillations, as a consequence of a homoclinic bifurcation, coexisting with homogeneous states are observed in several physical systems as lasers with saturable absorber [45], reaction-diffusion systems [27], seashells [46], catalytic reactions [47], and model of evolution of species [48]. Therefore, we expect that effects of local coupling in such frameworks can also generate chimera-type states.

It is important to note that coupling of oscillators with self-oscillation can exhibit the emergence of complex spatiotemporal behaviors such as phase turbulence without the need to be close to homoclinic bifurcation. If the system under study also coexists with a uniform state, then one would expect to observe chimera-type states.

The authors thank G. González and M. Wilson for fruitful discussions. M.G.C., M.A.G.-N., and R.G.R. acknowledge the financial support of FONDECYT Projects No. 1150507, No. 11130450, and No. 1130622, respectively. R.G.R., S.C., and M.A.G-N. thank financial support of ECOS-CONICYT project C15E06. 
[1] Y. Kuramoto, Chemical Oscillations, Waves, and Turbulence (Springer-Verlag, Berlin, 1984).

[2] A. Pikovsky, M. Rosenblum, J. Kurths, and R. C. Hilborn, Synchronization: A Universal Concept in Nonlinear Sciences (Cambridge University Press, Cambridge, UK, 2002).

[3] K. Kaneko and I. Tsuda, Chaos and Beyond: A Constructive Approach With Applications in Life Sciences (Springer, Berlin, 1996).

[4] B. I. Shraiman, A. Pumir, W. van Saarloos, P. C. Hohenberg, H. Chate, and M. Holen, Physica D 57, 241 (1992).

[5] A. C. Newell, D. A. Rand, and D. Russell, Physica D 33, 281 (1988).

[6] P. Coullet, L. Gil, and J. Lega, Phys. Rev. Lett. 62, 1619 (1989).

[7] H. Chate, Nonlinearity 7, 185 (1994).

[8] M. G. Clerc and N. Verschueren, Phys. Rev. E 88, 052916 (2013).

[9] D. M. Abrams and S. H. Strogatz, Phys. Rev. Lett. 93, 174102 (2004).

[10] Y. Kuramoto and D. Battogtokh, Nonlin. Phenom. Complex Syst. (Dordrecht, Neth.) 5, 380 (2002).

[11] D. M. Abrams and S. H. Strogatz, Int. J. Bifurcat. Chaos 16, 21 (2006); G. C. Sethia, A. Sen, and F. M. Atay, Phys. Rev. Lett. 100, 144102 (2008); O. E. Omel'chenko, M. Wolfrum, S. Yanchuk, Y. L. Maistrenko, and O. Sudakov, Phys. Rev. E 85, 036210 (2012); Y. L. Maistrenko, A. Vasylenko, O. Sudakov, R. Levchenko, and V. L. Maistrenko, Int. J. Bifurcat. Chaos 24, 1440014 (2014).

[12] S. Nkomo, M. R. Tinsley, and K. Showalter, Phys. Rev. Lett. 110, 244102 (2013); J. Hizanidis, V. Kanas, A. Bezerianos, and T. Bountis, Int. J. Bifurcat. Chaos 24, 1450030 (2014).

[13] C. R. Laing, Phys. Rev. E 81, 066221 (2010).

[14] I. Omelchenko, Y. L. Maistrenko, P. Hövel, and E. Schöll, Phys. Rev. Lett. 106, 234102 (2011).

[15] S. Olmi, A. Politi, and A. Torcini, Europhys. Lett. 92, 60007 (2010).

[16] I. Omelchenko, B. Riemenschneider, P. Hövel, Y. L. Maistrenko, and E. Schöll, Phys. Rev. E 85, 026212 (2012).

[17] D. M. Abrams, R. Mirollo, S. H. Strogatz, and D. A. Wiley, Phys. Rev. Lett. 101, 084103 (2008); C. R. Laing, Chaos 19, 013113 (2009); E. A. Martens, C. R. Laing, and S. H. Strogatz, Phys. Rev. Lett. 104, 044101 (2010); H. Wang and X. Li, Phys. Rev. E 83, 066214 (2011); T. Bountis, V. G. Kanas, J. Hizanidis, and A. Bezerianos, Eur. Phys. J. Spec. Top. 223, 721 (2014); L. Schmidt, K. Schönleber, K. Krischer, and V. Garcia-Morales, Chaos 24, 013102 (2014); A. Yeldesbay, A. Pikovsky, and M. Rosenblum, Phys. Rev. Lett. 112, 144103 (2014); J. Sieber, O. E. Omelchenko, and M. Wolfrum, ibid. 112, 054102 (2014).

[18] G. C. Sethia and A. Sen, Phys. Rev. Lett. 112, 144101 (2014).

[19] M. R. Tinsley, S. Nkomo, and K. Showalter, Nat. Phys. 8, 662 (2012).

[20] A. M. Hagerstrom, T. E. Murphy, R. Roy, P. Hövel, I. Omelchenko, and E. Schöll, Nat. Phys. 8, 658 (2012).

[21] E. A. Martens, S. Thutupalli, A. Fourriére, and O. Hallatschek, Proc. Natl. Acad. Sci. USA 110, 10563 (2013).

[22] G. C. Sethia, A. Sen, and G. L. Johnston, Phys. Rev. E 88, 042917 (2013).
[23] M. Wolfrum and O. E. Omel'chenko, Phys. Rev. E 84, 015201(R) (2011); M. Wolfrum, O. E. Omel'chenko, S. Yanchuk, and Y. L. Maistrenko, Chaos 21, 013112 (2011); G. Bordyugov, A. Pikovsky, and M. Rosenblum, Phys. Rev. E 82, 035205(R) (2010).

[24] S. Olmi, E. A Martens, S. Thutupalli, and A. Torcini, Phys. Rev. E 92, 030901 (2015); S. Olmi, Chaos 25, 123125 (2015).

[25] C. R. Laing, Phys. Rev. E 92, 050904(R) (2015).

[26] B.-W. Li and H. Dierckx, Phys. Rev. E 93, 020202(R) (2016).

[27] M. Argentina and P. Coullet, Physica A 257, 45 (1998).

[28] P. D. Woods and A. R. Champneys, Physica D 129, 147 (1999); P. Coullet, C. Riera, and C. Tresser, Phys. Rev. Lett. 84, 3069 (2000).

[29] R. I. Bogdanov, Funct. Anal. Appl. 9, 144 (1975); F. Takens, Comm. Math. Inst. Rijksuniv. Utrecht 3, 1 (1974), reprinted in, Chapter 1, Global Analysis of Dynamical Systems: Festschrift dedicated to Floris Takens for his 60th birthday, edited by H.W. Broer, B. Krauskopf, G. Vegter (IOP Publishing LTD, Bristol 2001).

[30] J. Claerbout, Fundamentals of Geophysical Data Processing (McGraw-Hill, New York, 1976); A. V. Oppenheim and R. W. Schafer, Digital Signal Processing (Prentice-Hall, Englewood Cliffs, NJ, 1975).

[31] J. Burke and E. Knobloch, Chaos 17, 037102 (2007).

[32] J. Burke and E. Knobloch, Phys. Rev. E 73, 056211 (2006); W. J. Firth, L. Columbo, and A. J. Scroggie, Phys. Rev. Lett. 99, 104503 (2007); U. Bortolozzo, M. G. Clerc, and S. Residori, Phys. Rev. E 78, 036214 (2008).

[33] F. Haudin, R. G. Rojas, U. Bortolozzo, S. Residori, and M. G. Clerc, Phys. Rev. Lett. 107, 264101 (2011).

[34] G. Fath, Physica D 116, 176 (1998).

[35] M. G. Clerc, R. G. Elias, and R. G. Rojas, Philos. Trans. R. Soc. A 369, 412 (2011).

[36] R. F. Peierls, Proc. Phys. Soc. 52, 34 (1940).

[37] F. R. N. Nabarro, Proc. Phys. Soc. 59, 256 (1947).

[38] F. R. N. Nabarro, Theory of Crystal Dislocations (Dover, New York, 1987).

[39] E. Ott, Chaos in Dynamical Systems, 2nd ed. (Cambridge University Press, Cambridge, UK, 2002).

[40] F. Christianen and H. H. Rough, Nonlinearity 10, 1063 (1997); T. J. Bridges and S. Reich, Physica D 156, 219 (2001).

[41] G. Nicolis, Introduction to Nonlinear Science (Cambridge University Press, Melbourne, 1995).

[42] N. Verschueren, U. Bortolozzo, M. G. Clerc, and S. Residori, Philos. Trans. R. Soc. A 372, 20140011 (2014).

[43] M. G. Clerc, C. Fernandez-Oto, M. A. García-Ñustes, and E. Louvergneaux, Phys. Rev. Lett. 109, 104101 (2012).

[44] N. Verschueren, U. Bortolozzo, M. G. Clerc, and S. Residori, Phys. Rev. Lett. 110, 104101 (2013).

[45] J. L. A. Dubbeldam and B. Krauskopf, Opt. Commun. 159, 325 (1999).

[46] D. R. Fowler, H. Meinhardt, and P. Prusinkiewicz, ACM SIGGRAPH Comput. Graph. 26, 379 (1992).

[47] V. Casagrande and A. S. Mikhailov, Physica D 205, 154 (2005).

[48] M. A. Bees, P. H. Coullet, and E. A. Spiegel, Chaos 18, 043114 (2008). 